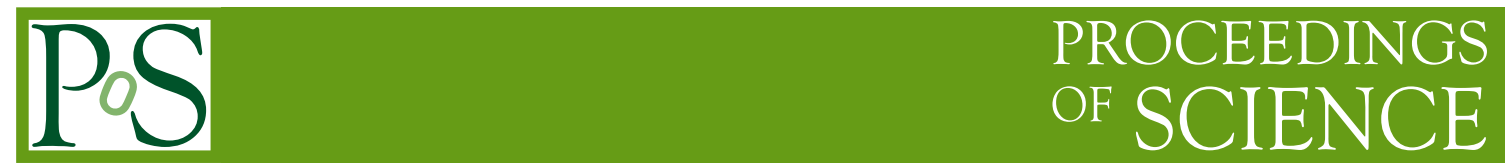

\title{
Making use of the International Lattice Data Grid
}

\author{
Tomoteru Yoshie* \\ Center for Computational Sciences, University of Tsukuba, Tsukuba 305-8577, Japan \\ E-mail: yoshiedccs.tsukuba.ac.jp
}

The International Lattice Data Grid (ILDG) continues stable operation for about one year and has accumulated a lot of valuable configurations. After a brief review of the ILDG system, we highlight large physics projects, whose configurations are already available on the grid or will be open to the public in the near future. With such information, one can make better use of the ILDG. Statistics about the ILDG is also reported.

The XXVI International Symposium on Lattice Field Theory

July 14 - 19, 2008

Williamsburg, Virginia, USA

${ }^{*}$ Speaker. 


\section{Introduction}

Six years ago at Lattice 2002 the construction of the International Lattice Data Grid was proposed [1]. After extensive work [2, 3, 4] by many people, the first stage system construction completed last year. The ILDG is already used to open data to the public and to share data within collaboration. With this situation in mind, we'd like to invite new users to the ILDG.

For this purpose, we give a brief system overview in sec. 2, describe how to use data on the grid in sec. 3, and highlight major ensembles on the grid in sec. 4, so that one can start to find interesting ensembles. We also give statistics about the ILDG in sec. 5.

\section{System overview}

The ILDG bears two aspects, metadata and middleware. Work on the ILDG has been carried out by the corresponding working groups. Current members of the working groups are

- Metadata working group (MDWG): P. Coddington (Adelaide), T. Yoshie (Tsukuba), D. Pleiter (DESY), G. Andronico (INFN), C. Maynard (Edinburgh), C. DeTar (Utah), J. Simone (FNAL), R. Edwards, B. Joo (JLAB)

- Middleware working group (MWWG): P. Coddington, S. Zhang (Adelade), T. Amagasa, N. Ishii. O. Tatebe. M. Sato (Tsukuba), D. Melkumyan, D. Pleiter (DESY), G. Beckett, R. Ostrowski (Edinburgh), J. Simone (FNAL), B. Joo, C. Watson (JLAB)

In addition, the ILDG board, which consists of one representative from each country, supervises two working groups and decides strategic issues. Current members are

- ILDG board: R. Brower (USA), K. Jansen (Germany), R. Kenway (UK, chair this year), D. Leinweber (Australia), O. Pene (France), F. Di Renzo (Italy), A. Ukawa (Japan)

Figure 1 sketches data and metadata components of the ILDG. Properties (metadata) of an ensemble such as physics parameters of the simulation and those of a configuration such as the trajectory number are marked up with the QCDml [3,5], an XML based markup language. The ensemble XML and the configuration XML are linked by a unique name of the ensemble called "markovChainURI". The configuration XML and a configuration file are linked by a unique name of the configuration called "dataLFN" (data Logical File Name).

Figure 2 summarizes middleware components of the ILDG and data-metadata flow [4]. Configurations are stored in "Storage Elements", while metadata are stored in the "Metadata Catalogue". Queries on ensemble and configuration metadata are made to the Metadata Catalogue. The "File Catalogue" maps the dataLFN to one or more locations for the file, or URLs of the file. After you get an URL, you can download the configuration file from one of storage elements. Authentication is made before you download. The VOMS (Virtual Organization Membership Service) server maintains the ILDG virtual organization (VO). The ILDG consists of five regional grids (RG), CSSM for Australia, JLDG for Japan, LDG (LatFor Data Grid) for continental Europe, UKQCD for UK, and USQCD for US. Implementations of storage elements and catalogue services are different grid by grid, but RGs are inter-operable with a common interface. 


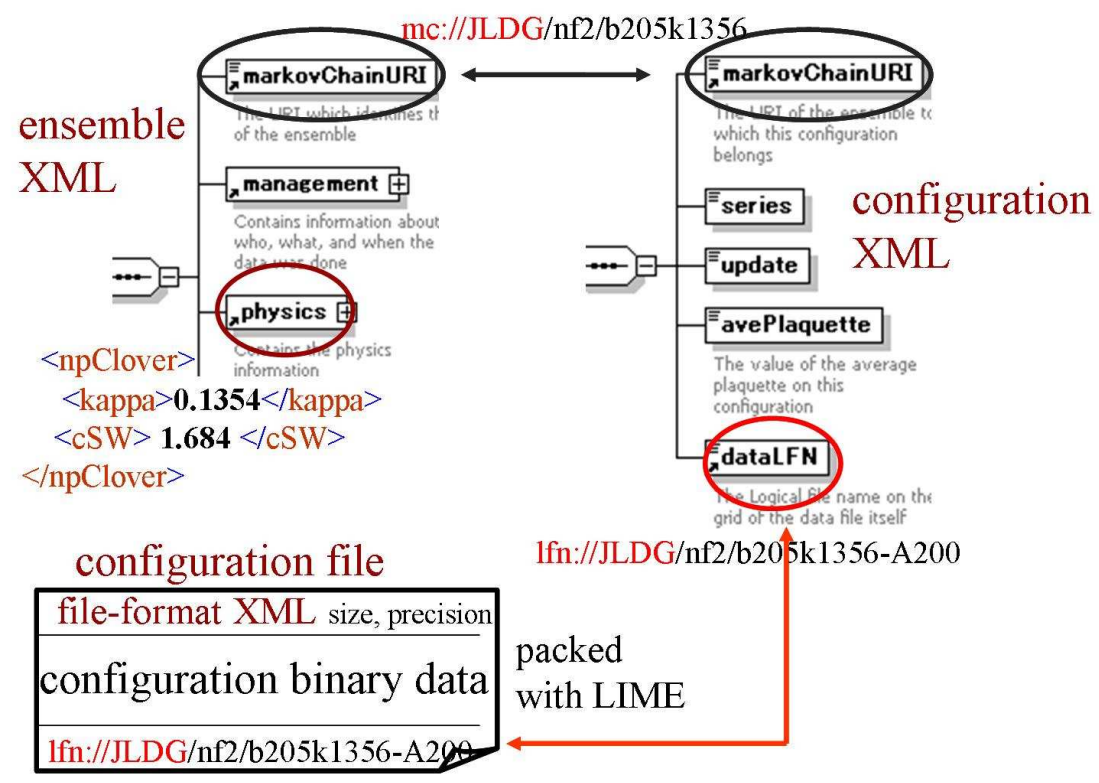

Figure 1: Metadata and data components and how they are linked.

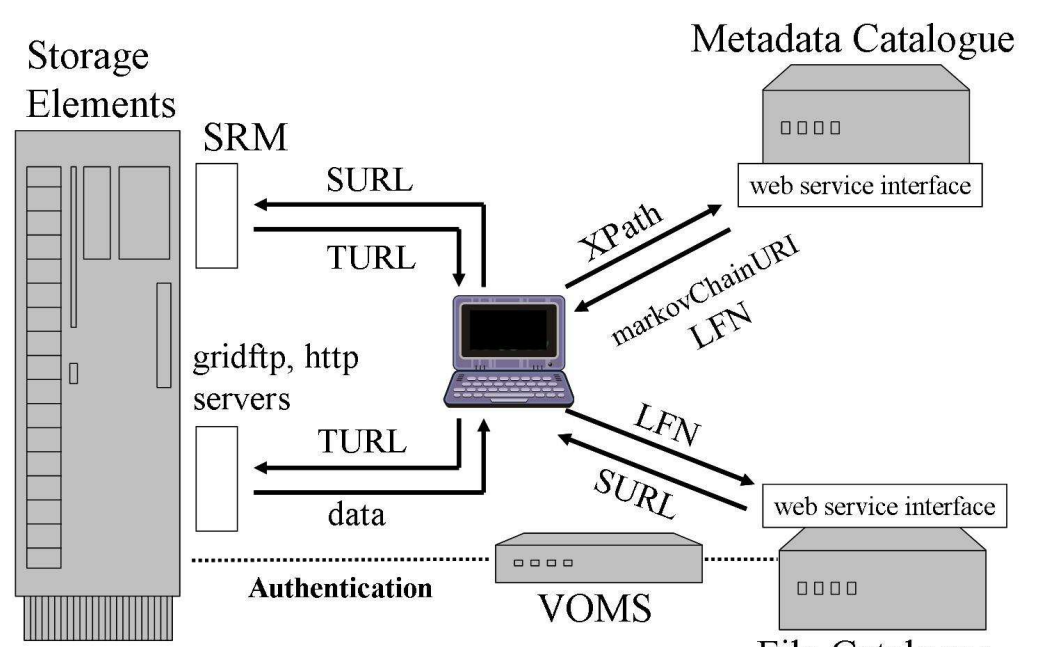

File Catalogue

Figure 2: Middleware components and data-metadata flow.

\section{Using data on ILDG}

Although the ILDG system is a little bit complicated, users don't have to remember details, because easy-to-use tools are developed and provided by the MWWG.

A procedure to use data on the ILDG is as follows.

1. Join the ILDG VO. To do this, one obtains a grid certificate from a CA (Certificate Authority) trusted by the IGTF (International Grid Trust Federation [6]), and visits the ILDG VOMRS 
(VO Member Registration Service [7]) to register. A representative of your regional grid will approve your registration request. The certificate is necessary when you download files.

2. Find interesting ensembles. You can use portals or tools provided by RGs, which can be accessed from the ILDG official web page [8]. Some details will be given below.

3. Check access policy before you use data. Data on the ILDG are either public or can be used after negotiation with the collaboration. The best way to know the policy is to contact the collaboration.

4. Download configurations. One can use a standard command line tool ildg-get provided by the MWWG. Note that some RGs support other methods to access data, such as ltools (LDG), DiGS tools (UKQCD) and uberftp (JLDG).

5. Do research and write a paper. Please acknowledge the collaboration by citing papers specified by the collaboration and the ILDG web page "http://www.lqcd.org/ildg/".

In addition to RG portals, a web page [9] prepared for the ILDG tutorial session of this conference, which is organized by C. Urbach and C. Allton, provides you with a good starting point.

In order to help users to find ensembles, RGs provide variety of data browsers and tools. The USQCD portal [10] and the LDG portal [11] show you a list of ensembles and details of each ensemble. The CSSM portal [12] enables you to search ensembles by specifying actions and other physics parameters. The UKQCD ildg-browser [13] supports semantic search based on XML. A new JLDG portal, which is still under development and will appear soon in [14], will support narrowing search by faceted navigation. Facets are categories of XML documents, such as names of regional grid, collaboration, project etc., and physics parameters. You will be able to specify any items in any order for narrowing candidates. See a screen shot in Fig. 3. You can use portals without joining the ILDG VO. Please visit and try all portals freely and find your favorite one.

\section{Ensembles on the grid}

This section summarizes major ensembles which are already available or will appear in the near future. We have asked several collaborations to list interesting ensembles, and have compiled replies. Therefore, what is shown below is not a complete list and may be biased due to our queries.

In the following tables, we use abbreviations for the action field, Sym: Symanzik, $L W$ : LuescherWeisz, $n p$ : non-perturbatively $O(a)$ improved, $t p$ : tadpole improved, and $T M$ : twisted mass. The number of configurations is approximate and the status field indicates status of ensembles by key words public: publicly available, negotiable: available but one can use them after negotiation with the collaboration, prod: : production run is on-going, and prep.: configurations are in preparation and will be available soon.

\subsection{CSSM}

Table 1 lists ensembles on the CSSM grid. The CSSM collaboration has started to accumulate $N_{f}=2$ configurations generated with the FLIC fermion action. We hear that they continue parameter tuning for a while and plan to quantify the advantages of the FLIC action for dynamical quark simulations. 


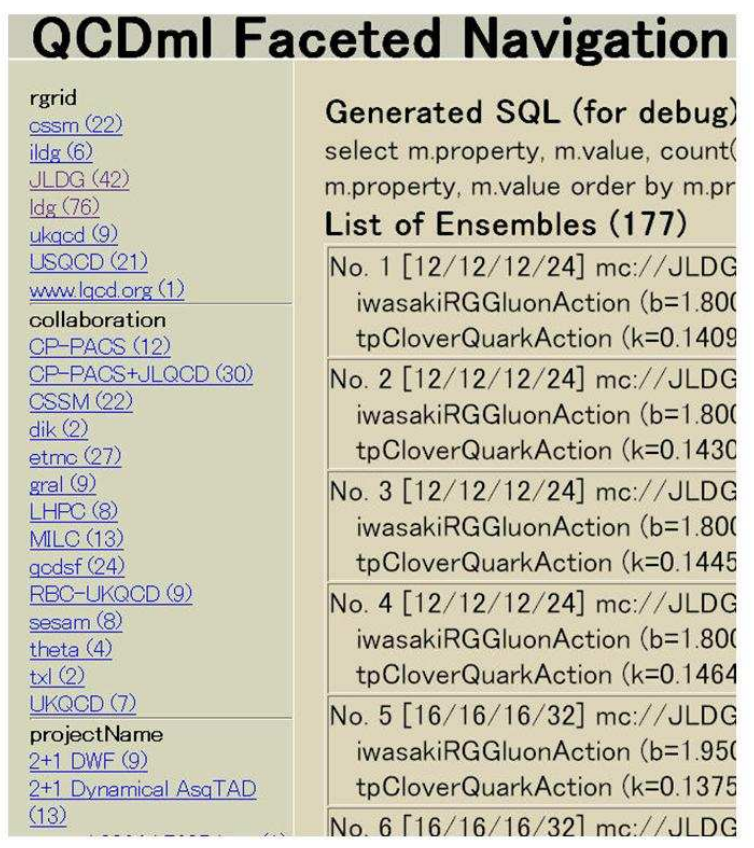

\section{QCDml Faceted Navig}

rgrid

JLDG (30)

collaboration

CP-PACS+JLQCD (30)

projectName

RCNF2+1 (NF=2+1 full QCD

with iwasaki RG gauge and

non-perturbatively $\mathrm{O}(\mathrm{a})$

improved wilson (olover)

quark action) (30)

date

$2007(30)$

size

$16 / 16 / 16 / 32(10)$

$20 / 20 / 20 / 40(10)$

$\frac{20 / 20 / 20 / 40(10)}{28 / 28 / 28 / 56(10)}$

numberOfflavours

$2+1(30)$

gluon

iwasakiRGGluonAction (30)

quark

npCloverQuarkAction (60)

beta

$1.8300000000(10)$

$1.90000000000(10)$

$20500000000(10)$
Generated SQL $(f$

select m.property, m.v (select $*$ from $\mathrm{mc}$ whe $\mathrm{c} 0$ on m.uri $=\mathrm{c} 0$.uri jo 'collaboration' and valc c1.uri group by m.prop List of Ensembles No. $1[16 / 16 / 16 / 32]$ $\mathrm{mc} / / / \mathrm{JLDG} / \mathrm{CP}-\mathrm{PAC}$ iwasakiRGGluonAct npCloverQuarkActic No. $2[16 / 16 / 16 / 32]$ $\mathrm{mc}: / / \mathrm{JLDG} / \mathrm{CP}-\mathrm{PAC}$ iwasakiRGGluonAct npCloverQuarkActic No. $3[16 / 16 / 16 / 32]$ $\mathrm{mc}: / / J L D G / C P-P A C$ iwasakiRGGluonAct npCloverQuarkActi No. $4[16 / 16 / 16 / 32]$

Figure 3: A screen shot of faceted navigation, taken from the new JLDG portal.

\begin{tabular}{c|c|c|c|c|c|c|c}
\hline \hline$N_{f}$ & action & collab & $a(\mathrm{fm})$ & lattice & $m_{\pi}(\mathrm{MeV})$ & conf & status \\
\hline 0 & $/$ tpLW,DBW2 & CSSM & & & & 1500 & public \\
\hline 2 & FLIC/tpLW & CSSM & 0.096 & $16^{3} \times 32$ & 820 & 50 & public \\
\hline 2 & FLIC/tpLW & CSSM & 0.125 & $20^{3} \times 40$ & $>300$ & & prod. \\
\hline \hline
\end{tabular}

Table 1: Major ensembles on the CSSM grid.

The CSSM grid has a 7 TB disk and 20 TB tape system for storage elements. Catalogue services are operated at CSSM.

\subsection{JLDG}

Table 2 shows ensembles on the JLDG. In addition to the CP-PACS $N_{f}=2$ and the CPPACS + JLQCD $N_{f}=2+1$ ensembles already public to the world, the $N_{f}=2$ and $N_{f}=2+1$ overlap quark ensembles and the $N_{f}=2+1$ npClover ensembles with very light quark masses will be publicly released by the JLQCD collaboration and the PACS-CS collaboration, respectively.

Storage elements, with 35 TB disk space in total, are distributed over six sites in Japan, Tsukuba, KEK, Kyoto, Osaka, Hiroshima and Kanazawa. Catalogue services are operated at CCS, Tsukuba.

\subsection{LDG}

For the LatFor data grid, ensembles generated by two major contributors are listed in table 3. The ETM collaboration has carried out $N_{f}=2$ simulations with the Wilson twisted mass quark 


\begin{tabular}{c|c|c|c|c|c|c|l}
\hline \hline$N_{f}$ & action & collab & $a(\mathrm{fm})$ & lattice & $m_{\pi}(\mathrm{MeV})$ & conf & status \\
\hline 2 & tpClover & CP-PACS & 0.22 & $12^{3} \times 24$ & $1060-490$ & $1000 \times 4$ & \multirow{2}{*}{ public } \\
\cline { 4 - 7 } & /Iwasaki & & 0.16 & $16^{3} \times 32$ & $1270-540$ & $1000 \times 4$ & \\
\cline { 4 - 7 } & & & 0.11 & $24^{3} \times 48$ & $1160-540$ & $800 \times 4$ & \\
\hline 2 & npClover/Plaq. & JLQCD & 0.09 & $20^{3} \times 48$ & $1370-600$ & $1200 \times 5$ & prep. $(* 1)$ \\
\hline $2+1$ & npClover & CP-PACS & 0.12 & $16^{3} \times 32$ & $1200-620$ & $800 \times 5 \times 2$ & \multirow{2}{*}{ public } \\
\cline { 4 - 7 } & /Iwasaki & + JLQCD & 0.10 & $20^{3} \times 40$ & $1100-650$ & $800 \times 5 \times 2$ & \\
\cline { 4 - 7 } & & & 0.07 & $28^{3} \times 56$ & $1030-630$ & $600 \times 5 \times 2$ & \\
\hline 2 & Overlap/Iwasaki & JLQCD & 0.12 & $16^{3} \times 32$ & $750-290$ & $500 \times 6$ & prep. $(* 1)$ \\
\hline $2+1$ & Overlap/Iwasaki & JLQCD & 0.11 & $16^{3} \times 48$ & $800-310$ & $500 \times 5 \times 2$ & $(* 2)$ \\
\hline $2+1$ & npClover & PACS-CS & 0.09 & $32^{3} \times 64$ & $702-156$ & $400 \times 4$ & \multirow{2}{*}{$(* 3)$} \\
& $/$ Iwasaki & & & & & $800 \times 2$ & \\
\hline \hline
\end{tabular}

Table 2: Major ensembles on the JLDG. $(* 1$ : will be public, $* 2$ : available date not decided yet, $* 3$ will be public 6 months after a spectrum paper is submitted.)

action for three lattice spacings and for a wide range of quark masses. All of these configurations are already put on the grid and can be used after negotiation with the collaboration. We hear that they will become publicly available probably by the end of this year. The collaboration has also started $N_{f}=2+1+1$ simulations. $N_{f}=2$ configurations from the QCDSF collaboration, also cover wide ranges of lattice spacings and quark masses, need negotiation. $N_{f}=2+1$ simulations with the SLiNC quark action is on-going. The LDG contains data from other collaborations such as SESAM, T $\chi \mathrm{L}$, GRAL, DIK, and Theta. We hear that the ALPHA collaboration has no plan to submit data, and the Bern-Marseilles-Wuppertal collaboration has not made a decision about their plans for the ILDG.

The LatFor data grid is for continental Europe. Storage elements are distributed over 3 sites in Germany, DESY (Hamburg+Zeuthen), JSC (Jülich), ZIB (Berlin), CC-IN2P3 in Lyon, France and INFN Parma in Italy. Storage elements have tape back-end without fixed storage quota. The RG operates catalogue services at DESY.

\subsection{UKQCD}

The UKQCD grid (see table 4) contains $N_{f}=2+1$ domain wall ensembles generated by the joint collaboration of the UKQCD and the RBC. $16^{3}$ lattices were publicly available before this conference. The collaboration has publicly released configurations on $24^{3}$ lattices this August. Simulations on finer lattices are on-going. The grid has also archived the UKQCD asqtad lattices. Configurations on the coarser lattice are publicly available, while those on the finer lattice are negotiable.

The UKQCD grid consists of seven sites in UK, Edinburgh, ACF (U. of Edinburgh), Glasgow, Liverpool, Swansea, RAL Didcot and Southampton. The grid maintains 80 TB disk space (as of March 2007) for storage elements. Catalogue services are operated at EPCC, Edinburgh. 


\begin{tabular}{c|c|c|c|c|c|c|l}
\hline \hline$N_{f}$ & action & collab & $a(\mathrm{fm})$ & lattice & $m_{\pi}(\mathrm{MeV})$ & conf & status \\
\hline 2 & wilson-TM & ETM & 0.100 & $20^{3} \times 48$ & $700-300$ & $2000 \times 4$ & \multirow{2}{*}{$(*$ negotiable } \\
& /Sym & & & $24^{3} \times 48$ & $700-300$ & $2000 \times 5$ & \multirow{2}{*}{$(1)$} \\
& & & 0.085 & $24^{3} \times 48$ & $700-300$ & $2500 \times 5$ & \\
& & & & $32^{3} \times 64$ & $300-250$ & $2500 \times 2$ & \\
\cline { 3 - 7 } & & & 0.066 & $20^{3} \times 48$ & $400-280$ & $3000 \times 2$ & \\
& & & $24^{3} \times 48$ & 350 & 3000 & \\
\hline $2+1+1$ & wilson-TM & ETM & 0.090 & $24^{3} \times 48$ & $700-300$ & O $(1000)$ & prod. \\
& /Sym & & & & & & \\
\hline 2 & npClover & QCDSF & 0.11 & $16^{3} \times 32$ & $1200-250$ & 19 ensembles & negotiable \\
& /wilson & & -0.07 & $-40^{3} \times 64$ & & $\approx 20000(* 2)$ & \\
\hline $2+1$ & SLiNC & QCDSF & 0.08 & $48^{3} \times 64$ & $500-200$ & & prod. \\
& /Sym & & & & & & \\
\hline \hline
\end{tabular}

Table 3: Major ensembles on the LDG. $(* 1$ : become publicly available probably by the end of $2008, * 2$ : based on Metadata Catalogue)

\begin{tabular}{c|c|c|c|c|c|c|l}
\hline \hline$N_{f}$ & action & collab & $a(\mathrm{fm})$ & lattice & $m_{\pi}(\mathrm{MeV})$ & conf & status \\
\hline $2+1$ & Domain Wall & UKQCD & 0.12 & $16^{3} \times 32 \times 16$ & 630 & 1517 & public \\
& /Iwasaki & /RBC & & & 530 & 810 & \\
& & & & 400 & 832 & \\
\cline { 4 - 8 } & & & 0.12 & $24^{3} \times 64 \times 16$ & $670-330$ & $800 \times 4$ & public $(* 1)$ \\
\cline { 4 - 8 } & & & 0.08 & $32^{3} \times 64 \times 16$ & $400-280$ & & prod. \\
& & & $48^{3} \times 64 \times 16$ & $\approx 220$ & & \\
\hline $2+1$ & \multirow{2}{*}{ asqtad/tpSym } & \multirow{2}{*}{ UKQCD } & 0.12 & $24^{3} \times 64$ & 290 & 5081 & public \\
\cline { 3 - 8 } & & & 0.09 & $32^{3} \times 64$ & 360 & 700 & negotiable \\
\hline \hline
\end{tabular}

Table 4: Major ensembles on the UKQCD grid. (*1: public release in this August)

\subsection{USQCD}

Table 5 summarizes ensembles in the USQCD grid. The MILC collaboration has been generating an extensive set of $N_{f}=2+1$ ensembles using the asqtad quark action. A remarkable point is that the collaboration makes all data public as soon as they are created. Currently, they are generating data on large lattices at 0.12 and $0.09 \mathrm{fm}$, and on much finer lattices. They will be available on the grid or the NERSC Gauge Connection web site [15]. The LHP collaboration is generating anisotropic lattices. $N_{f}=2$ data are already publicly available, and $N_{f}=2+1$ data are coming soon.

Storage elements of this grid are operated at Fermilab as a part of huge resources, while catalogue services are operated at JLAB. 


\begin{tabular}{|c|c|c|c|c|c|c|c|}
\hline$N_{f}$ & action & collab & $a(\mathrm{fm})$ & lattice & $m_{\pi}(\mathrm{MeV})$ & conf & status \\
\hline \multirow[t]{7}{*}{$2+1$} & \multirow{7}{*}{$\begin{array}{l}\text { asqtad } \\
/ \text { tpLW }\end{array}$} & \multirow[t]{7}{*}{ MILC } & 0.15 & $(16-20)^{3} \times 48$ & $711-235$ & $600 \times 4$ & public \\
\hline & & & \multirow[t]{2}{*}{0.12} & $(20-24)^{3} \times 64$ & $500-260$ & $1700 \times 4$ & public \\
\hline & & & & $32^{3} \times 64$ & $\approx 260$ & & prod. \\
\hline & & & \multirow[t]{2}{*}{0.09} & $(28-40)^{3} \times 96$ & $480-240$ & $1100 \times 6$ & public, prod. \\
\hline & & & & $40^{3} \times 96$ & $\approx 240$ & & prod. \\
\hline & & & 0.06 & $(48-64)^{3} \times 144$ & $430-220$ & $600 \times 4$ & public, prod. \\
\hline & & & 0.045 & $64^{3} \times 192$ & TBD. & 300 & public, prod. \\
\hline \multirow[t]{3}{*}{2} & \multirow{3}{*}{$\begin{array}{l}\text { aniso. } \\
\text { wilson } \\
\text { /wilson }\end{array}$} & \multirow[t]{3}{*}{ LHP } & \multirow[t]{3}{*}{0.11} & $16^{3} \times 64$ & 600 & 861 & \multirow[t]{3}{*}{ public } \\
\hline & & & & $24^{3} \times 64$ & 600 & 871 & \\
\hline & & & & & 440 & 1535 & \\
\hline $2+1$ & $(* 1)$ & LHP & 0.12 & $24^{3} \times 128$ & 330 & 2000 & prep. \\
\hline
\end{tabular}

Table 5: Major ensembles on the USQCD grid. (*1: aniso. clover/ tadpole improved Symanzik with no rectangle loops in temporal direction.)

\begin{tabular}{l||r|r|r|r}
\hline \hline RG & \#VO member & \#ensemble & \#config. & data size (TB) \\
\hline CSSM & 7 & 22 & $1.7 \mathrm{~K}$ & 0.1 \\
JLDG & 4 & 42 & $29.2 \mathrm{~K}$ & 7.5 \\
LDG & 49 & 82 & $142.6 \mathrm{~K}$ & 28.0 \\
UKQCD & 26 & 16 & $8.8 \mathrm{~K}$ & 2.9 \\
USQCD & 7 & 21 & $10.9 \mathrm{~K}$ & 2.7 \\
\hline total & 93 & 183 & $193 \mathrm{~K}$ & 41 \\
\hline \hline
\end{tabular}

Table 6: Statistics about the ILDG as of June 26, 2008. Data are taken from VOMRS and Metadata Catalogues. Data size does not include file replica.

\section{Statistics}

In order to see how the ILDG is utilized, statistics about the ILDG is summarized in table 6.

We have 93 ILDG VO members in total. Because the LDG and the UKQCD grid have many users, we suppose that they use the ILDG as their primary storage infrastructure. The CSSM grid and the USQCD grid have genuine users. The JLDG has only admin users. We hope may Japanese users, who still use an old system, will move to the ILDG.

Number of ensembles stored in the ILDG increases almost linearly since January 2006 and have reached 183 . We currently have $\approx 190 \mathrm{~K}$ configurations with total size of $\approx 40 \mathrm{~TB}$.

\section{Conclusions and future work}

The ILDG continues stable operation and has already accumulated a lot of valuable configurations. Usability of the ILDG is improved significantly. The ILDG is becoming an important research infrastructure in this community. We hope that many more users join the ILDG and make better use of archived data for physics researches. 
In this report, we have described how to use data on the ILDG. Submitting data to the ILDG is a somewhat complicated procedure. Working group members think that making the procedure easy is an important future direction. Two working groups are also discussing extensions and improvements of the system, such as quark propagator sharing (MDWG) and replication of data among regional grids (MWWG).

I am grateful to all members of the ILDG working groups and the ILDG board, in particular to G. Beckett, C. DeTar, and D. Pleiter for helpful suggestions on the manuscript. I also thank colleagues who provided us with ensemble information on each regional grid. A part of this work is supported by the Grant-in-Aid of the Ministry of Education (No. 18104005 ) of the Japanese Government.

\section{References}

[1] C. T. H. Davies, A. C. Irving, R. D. Kenway and C. M. Maynard [UKQCD collaboration], International Lattice Data Grid, Nucl. Phys. (Proc. Suppl.) 119 (2003) 225 [hep-lat/ 0209121 ].

[2] A. C. Irving, R .D. Kenway, C. M. Maynard and T. Yoshie, Progress in building an International Lattice Data Grid, Nucl. Phys. (Proc.Suppl.) 129 (2004) 159 [hep-lat / 030902 9];

A. Ukawa, Status of International Lattice Data Grid - An Overview -, Nucl. Phys. (Proc. Suppl.) 140 (2005) 207 [hep-lat/ 0409084 ];

K. Jansen, Status Report on ILDG activities, PoS(LAT2006)013 [hep-lat / 0609012 ];

C. DeTar, Sharing Lattices Throughout the World: An ILDG Status Report, PoS(LATTICE 2007)009 [arXiv: 0710.1660 (hep-lat)].

[3] T. Yoshié et al. [ILDG Metadata Working Group], Marking up lattice QCD configurations and ensembles, PoS(LATTICE 2007)048 [arXiv: 0710.0230 (hep-lat)], and references therein.

[4] D. Pleiter et al. [ILDG Middleware Working Group], Towards an interoperable International Lattice Datagrid, PoS(LATTICE 2007)044 [arXiv: 0711.4852 (hep-lat)], and references therein.

[5] http://www.ccs.tsukuba.ac.jp/ILDG/

[6] http://www.igtf.net/

[7] https://grid-voms.desy.de:8443/vo/ildg/vomrs

[8] http://www.lqcd.org/ildg/

[9] http://people.physik.hu-berlin.de/ urbach/ildg-2.html (This page may be moved to the ILDG web site [8].)

[10] http://www.usqcd.org/ildg/

[11] http://www-zeuthen.desy.de/latfor/ldg/

[12] http://cssm.sasr.edu.au/ildg/

[13] http://www.gridpp.ac.uk/qcdgrid/

[14] http://www.jldg.org/

[15] http://qcd.nersc.gov/ 